\title{
Stochastic resonance as a collective property of ion channel assemblies
}

\author{
G. Schmid, I. Goychuk, and P. Hänggi \\ University of Augsburg, Department of Physics, D-86135 Augsburg, Germany
}

October 30, 2018

\begin{abstract}
By use of a stochastic generalization of the Hodgkin-Huxley model we investigate both the phenomena of stochastic resonance (SR) and coherence resonance (CR) in variable size patches of an excitable cell membrane. Our focus is on the challenge how internal noise stemming from individual ion channels does affect collective properties of the whole ensemble. We investigate both an unperturbed situation with no applied stimuli and one in which the membrane is stimulated externally by a periodic signal and additional external noise. For the nondriven case, we demonstrate the existence of an optimal size of the membrane patch for which the internal noise causes a most regular spike activity. This phenomenon shall be termed intrinsic CR. In presence of an applied periodic stimulus we demonstrate that the signal-to-noise ratio (SNR) exhibits SR vs. decreasing patch size, or vs. increasing internal noise strength, respectively. Moreover, we demonstrate that conventional SR vs. the external noise intensity occurs only for sufficiently large membrane patches, when the intensity of internal noise is below its optimal level. Thus, biological SR seemingly is rooted in the collective properties of large ion channel ensembles rather than in the individual stochastic dynamics of single ion channels.
\end{abstract}

PACS number(s): 87.10.+e, 87.16.-b, 05.40.-a

During the last decade, the effect of stochastic resonance (SR) - a cooperative phenomenon wherein the addition of external noise improves the detection and transduction of signals in nonlinear systems (for a comprehensive survey and prominent references, see Ref. [1] - has been studied experimentally and theoretically in various biological systems [2, 3, 4, 5, 6]. For example, SR has been experimentally demonstrated within the mechanoreceptive system in crayfish [2], in the cricket cercal sensory system [3], for human tactile sensation [4], visual perception [5], and response behavior of the arterial baroreflex system of humans [6]. The importance of this SR-phenomenon for sensory biology is by now well established; yet, it is presently not known to which minimal level of the biological organization the stochastic resonance effect can ultimately be traced down. Presumably, SR has its origin in the stochastic properties of the 
ion channel clusters located in a receptor cell membrane. Indeed, for an artificial model system Bezrukov and Vodyanoy have demonstrated experimentally that a large parallel ensemble of the alamethicin ion channels does exhibit stochastic resonance [7]. This in turn provokes the question whether a single ion channel is able to exhibit SR, or whether stochastic resonance is the result of a collective response from a finite assembly of channels. Stochastic resonance in single, biological potassium ion channels has also been investigated both theoretically [8] and experimentally [9]. This very experiment did not convincingly exhibit SR in single voltage-sensitive ion channels. Nevertheless, the SR phenomenon can occur in a single ion channel if only the parameters are within a regime where the channel is predominantly dwelled in the closed state, as demonstrated within a theoretical modeling for a potassium Shaker channel [B]. This prominent result, i.e. the manifestation of SR on the single-molecular level, is not only of academic interest, but is relevant also for potential nanotechnological applications, such as the design of single-molecular biosensors. The origin and biological relevance of SR in single ion channels, however, remains still open. Indeed, biological SR can be a manifestation of collective properties of large assemblies of ion channels of different sorts. To display the phenomenon of excitability these assemblies must contain a collection of ion channels of at least two different kinds - such as, e.g., potassium- and sodium-channels. The corresponding mean-field type model has been put forward by Hodgkin and Huxley in 1952 10 by neglecting the mesoscopic fluctuations which originate from the stochastic opening and closing of channels. SR due to external noise in this primary model and related models of excitable dynamics has extensively been addressed [11]. These models further display another interesting effect in the presence of noise, namely so termed coherence resonance (CR) [12]: even in absence of an external periodic signal the stochastic dynamics exhibits a surprisingly more regular behavior solely due to an optimally applied external noise intensity. A challenge though still remains: Does internal noise play a constructive role for SR and CR? Internal noise is produced by fluctuations of individual channels within the assembly, and diminishes with increasing number. For a large, macroscopic number of channels this noise becomes negligible. Under the realistic biological conditions, however, it may play a crucial role.

Our starting point is due to the model of Hodgkin and Huxley [10], i.e. the ion current across the biological membrane is carried mainly by the motion of sodium, $\mathrm{Na}^{+}$, and potassium, $\mathrm{K}^{+}$, ions through selective and voltage-gated ion channels embedded across the membrane. Besides, there is also a leakage current present which is induced by chloride and remaining other ions. The ion channels are formed by special membrane proteins which undergo spontaneous, but voltage-sensitive conformational transitions between open and closed states [13]. Moreover, the conductance of the membrane is directly proportional to the number of the open ion channels. This number depends on the potential difference across the membrane, $V$. The different concentrations of the ions inside and outside the cell are encoded by corresponding reversal potentials $E_{\mathrm{Na}}=50 \mathrm{mV}, E_{K}=-77 \mathrm{mV}$ and $E_{\mathrm{L}}=-54.4 \mathrm{mV}$, respectively, which give the voltage values at which the direction of partial ion currents is reversed [13]. 
Taking into account that the membrane possesses a capacitance $C=1 \mu \mathrm{F} / \mathrm{cm}^{2}$, Kirchhoff's first law reads in presence of an external current $I_{\text {ext }}(t)$ stimulus:

$$
C \frac{d}{d t} V+G_{\mathrm{K}}(n)\left(V-E_{\mathrm{K}}\right)+G_{\mathrm{Na}}(m, h)\left(V-E_{\mathrm{Na}}\right)+G_{\mathrm{L}}\left(V-E_{L}\right)=I_{\mathrm{ext}}(t) .
$$

Here, $G_{\mathrm{Na}}(m, h), G_{\mathrm{K}}(n)$ and $G_{\mathrm{L}}$ denote the conductances of sodium, potassium, and the remaining other ion channels, respectively. The leakage conductance is assumed to be constant, $G_{\mathrm{L}}=0.3 \mathrm{mS} / \mathrm{cm}^{2}$; in contrast, those of sodium and potassium depend on the probability to find the ion channels in their open conformation. To explain the experimental data, Hodgkin and Huxley did assume that the conductance of a potassium channel is gated by four independent and identical gates. Thus, if $n$ is the probability of one gate to be open, the probability of the $\mathrm{K}^{+}$channel to stay open is $P_{\mathrm{K}}=n^{4}$. Moreover, the gating dynamics of sodium channel is assumed to be governed by three independent, identical gates with opening probability $m$ and an additional one, being different, possessing the opening probability $h$. Accordingly, the opening probability of $\mathrm{Na}^{+}$ channel (or the fraction of open channels) reads $P_{\mathrm{Na}}=m^{3} h$. The conductances for potassium and sodium thus read

$$
G_{\mathrm{K}}(n)=g_{\mathrm{K}}^{\max } n^{4}, \quad G_{\mathrm{Na}}(m, h)=g_{\mathrm{Na}}^{\max } m^{3} h,
$$

where $g_{\mathrm{Na}}^{\max }=120 \mathrm{mS} / \mathrm{cm}^{2}$ and $g_{\mathrm{K}}^{\max }=36 \mathrm{mS} / \mathrm{cm}^{2}$ are the maximal conductances. Furthermore, the gating variables (probabilities) $m, h$ and $n$ obey the two-state, "opening-closing" dynamics,

$$
\begin{aligned}
\dot{m} & =\alpha_{m}(V)(1-m)-\beta_{m}(V) m, \\
\dot{h} & =\alpha_{h}(V)(1-h)-\beta_{h}(V) h, \\
\dot{n} & =\alpha_{n}(V)(1-n)-\beta_{n}(V) n,
\end{aligned}
$$

with the experimentally determined voltage-dependent transition rates, reading [10, 14]

$$
\begin{array}{ll}
\alpha_{m}(V)=\frac{0.1(V+40)}{1-\exp [-(V+40) / 10]}, & \beta_{m}(V)=4 \exp [-(V+65) / 18], \\
\alpha_{h}(V)=0.07 \exp [-(V+65) / 20], & \beta_{h}(V)=\{1+\exp [-(V+35) / 10]\}^{-1}, \\
\alpha_{n}(V)=\frac{0.01(V+55)}{1-\exp [-(V+55) / 10]}, & \beta_{n}(V)=0.125 \exp [-(V+65) / 80] .
\end{array}
$$

These nonlinear Hodgkin-Huxley equations (11)-(位) present a cornerstone model in neurophysiology. Within the same line of reasoning this model can be generalized to a mixture of different ion channels with various gating properties [14. 15. An essential drawback of this model, however, is that it operates with 
the average number of open channels, thereby disregarding corresponding number fluctuations (or, the so-called channel noise [16]). Thus, it can strictly be valid only within the limit of very large system size. We emphasize, however, that the size of an excitable membrane patch within a receptor cell is realistically finite. As a consequence, the role of internal fluctuations cannot be $a$ priori neglected; as a matter of fact, as shown below, they do play a key role for SR and CR.

The role of channel noise for the neuron firing has been studied by Lecar and Nossal as early as in 1971 [17]. The corresponding stochastic generalizations of Hodgkin-Huxley model (within a kinetic model which corresponds to the above given description) has been put forward by DeFelice et al. [18] and others; see [16] for a review and further references therein. The main conclusion of these previous studies is that the channel noise can be functionally important for neuron dynamics. It particular, it has been demonstrated that channel noise alone can give rise to a spiking activity even in the absence of any stimulus [16, 18].

The stochastic kinetic scheme [16, 18], however, necessitates extensive numerical simulations [19]. To aim at a less cumbersome numerical scheme we use a short-cut procedure that starts from Eq. (3) in order to derive a corresponding set of Langevin equations for a stochastic generalization of the Hodgkin-Huxley equations of the type put forward by Fox and $\mathrm{Lu}$ [20]. Following their reasoning we substitute the equations (3) with the corresponding Langevin generalization:

$$
\begin{aligned}
\dot{m} & =\alpha_{m}(V)(1-m)-\beta_{m}(V) m+\xi_{m}(t), \\
\dot{h} & =\alpha_{h}(V)(1-h)-\beta_{h}(V) h+\xi_{h}(t), \\
\dot{n} & =\alpha_{n}(V)(1-n)-\beta_{n}(V) n+\xi_{n}(t),
\end{aligned}
$$

with independent Gaussian white noise sources of vanishing mean. The noise autocorrelation functions depend on the stochastic voltage and the corresponding total number of ion channels as follows:

$$
\begin{aligned}
\left\langle\xi_{m}(t) \xi_{m}\left(t^{\prime}\right)\right\rangle & =\frac{2}{N_{\mathrm{Na}}} \frac{\alpha_{m} \beta_{m}}{\left(\alpha_{m}+\beta_{m}\right)} \delta\left(t-t^{\prime}\right), \\
\left\langle\xi_{h}(t) \xi_{h}\left(t^{\prime}\right)\right\rangle & =\frac{2}{N_{\mathrm{Na}}} \frac{\alpha_{h} \beta_{h}}{\left(\alpha_{h}+\beta_{h}\right)} \delta\left(t-t^{\prime}\right), \\
\left\langle\xi_{n}(t) \xi_{n}\left(t^{\prime}\right)\right\rangle & =\frac{2}{N_{\mathrm{K}}} \frac{\alpha_{n} \beta_{n}}{\left(\alpha_{n}+\beta_{n}\right)} \delta\left(t-t^{\prime}\right) .
\end{aligned}
$$

In order to confine the conductances between the physically allowed values between 0 (all channels closed) and $g^{\max }$ (all channels open) we have implemented numerically the constraint of reflecting boundaries so that $m(t), h(t)$ and $n(t)$ are always located between zero and one [20].

Moreover, the numbers $N_{\mathrm{Na}}$ and $N_{\mathrm{K}}$ depend on the actual area $S$ of the membrane patch. With the assumption of homogeneous ion channels densities, 
$\rho_{\mathrm{Na}}=60 \mu \mathrm{m}^{2}$ and $\rho_{\mathrm{K}}=18 \mu \mathrm{m}^{2}$, the following scaling behavior follows:

$$
N_{\mathrm{Na}}=\rho_{\mathrm{Na}} S, \quad N_{\mathrm{K}}=\rho_{\mathrm{K}} S .
$$

Upon decreasing the system size $S$, the fluctuations and, hence, the internal noise increases.

Before integrating the system of stochastic equations (11), (5), (6) numerically, the external stimulus $I_{\text {ext }}(t)$ must be specified. We take a periodic stimulus of the form

$$
I_{\text {ext }}(t)=A \sin (\Omega t)+\eta(t),
$$

where the sinusoidal signal with amplitude $A$ and frequency $\Omega$ is contaminated by the Gaussian white noise $\eta(t)$ with the autocorrelation function

$$
\left\langle\eta(t) \eta\left(t^{\prime}\right)\right\rangle=2 D_{\text {ext }} \delta\left(t-t^{\prime}\right),
$$

and noise strength $D_{\text {ext }}$. The numerical integration is carried out by the standard Euler algorithm with the step size $\Delta t \approx 2 \cdot 10^{-3} \mathrm{~ms}$. The "Numerical Recipes" routine ran2 is used for the generation of independent random numbers [21 with the Box-Muller algorithm providing the Gaussian distributed random numbers. The total integration time is chosen to be a multiple of the driving period $T_{\Omega}=2 \pi / \Omega$, as to ensure that the spectral line of the driving signal is centered on a computed value of the power spectral densities. From the stochastic voltage signal $V(t)$ we extract a point process of spike occurrences $\left\{t_{i}\right\}$ :

$$
u(t):=\sum_{i=1}^{N} \delta\left(t-t_{i}\right),
$$

where $N$ is the total number of spikes occurring during the elapsed time interval. The occurrence of a spike in the voltage signal $V(t)$ is obtained by upwardcrossing a certain detection threshold value $V_{0}$. It turns out that the threshold can be varied over a wide range with no effect on the resulting spike train dynamics. However, to account for the typical spike duration a time interval of $2 \mathrm{~ms}$ has been used. The power spectral density of the spike train $\left(\mathrm{PSD}_{u}\right)$, the interspike interval histogram (ISIH) and the coefficient of variation (CV) have been analyzed. The coefficient of variation $\mathrm{CV}$, which presents a measure of the spike coherence, reads:

$$
\mathrm{CV}:=\frac{\sqrt{\left\langle T^{2}\right\rangle-\langle T\rangle^{2}}}{\langle T\rangle}
$$

where $\langle T\rangle:=\lim _{N \rightarrow \infty} \frac{1}{N} \sum\left(t_{i+1}-t_{i}\right)$ and $\left\langle T^{2}\right\rangle:=\lim _{N \rightarrow \infty} \frac{1}{N} \sum\left(t_{i+1}-t_{i}\right)^{2}$ are the mean and mean-squared interspike intervals, respectively. From the $\mathrm{PSD}_{u}$ we obtain the height of the spectral line of the periodic stimulus as the difference between the peak value and its background offset. The signal-to-noise 

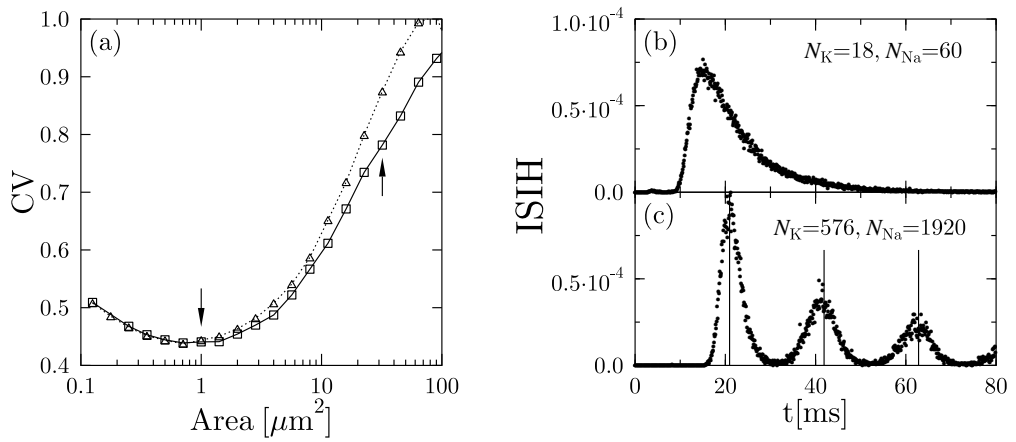

Figure 1: The CV defined in equation (11) plotted versus area $S$ (a) for $A=1.0 \mu \mathrm{A} / \mathrm{cm}^{2}, \Omega=0.3 \mathrm{~ms}^{-1}$, in the absence of external noise $D_{\text {ext }}=0$ (solid line) and with no external stimulus applied (dotted line). The ISIH are depicted in the presence of signal for $S=1 \mu \mathrm{m}^{2}$ (b), and $S=32 \mu \mathrm{m}^{2}$ (c). The vertical lines denote the driving period and the first two multiples.

ratio (SNR) is then given by the ratio of signal peak height to the background height (in the units of spectral resolution of signals).

We have analyzed the spike coherence in the autonomous, nondriven regime (i.e., we use $I_{\text {ext }}=0$ ) as a function of the decreasing cluster size. Our simulation reveals, cf. fig. 1 1 (a), the novel phenomenon of intrinsic coherence resonance, where the order in the spike sequence increases; i.e. the CV is decreasing, solely due to the presence of internal noise. The fully disordered sequence (which corresponds to a Poissonian spike train) would assume the value $\mathrm{CV}=1$. We note, however, that near $S=1 \mu \mathrm{m}^{2}$ (optimal dose of internal noise), CV $\approx 0.44$, i.e. the spike train becomes distinctly more ordered! For $S<1 \mu \mathrm{m}^{2}$, the internal noise increases further beyond the optimal value and destroys the order in spiking again. It is worth noting that for $S<1 \mu \mathrm{m}^{2}$ the model reaches limiting validity; in that regime the kinetic scheme [16, 18, 19] should be used instead. Such a corresponding study, however, has been put forward independently by Jung and Shuai [19]; their results are in qualitative agreement with our findings. Next we switch on an external sinusoidal driving: Interestingly enough the interspike intervals distribution is not affected for small patch sizes, cf. fig. 11(b) for $S=1 \mu \mathrm{m}^{2}$. In this case, the spike-activity possesses an internal rhythm which dominates over the external disturbances. For larger patch sizes the internal noise decreases and the periodic drive induces a reduction of the $\mathrm{CV}$ as compared to the undriven case, note the right arrow in fig. I1(a). In this latter regime the external driving rules the spiking activity as depicted with the characteristic peaks in the ISIH in fig. 11(c) at multiple driving periods.

Next, the focus is on the SNR in absence of external noise, see fig. 2(a). Here we discover the novel effect of genuine intrinsic stochastic resonance, where the response of the system to the external stimulus is optimized solely due to internal, omnipresent noise. For the given parameters it occurs at $S \approx 32 \mu \mathrm{m}^{2}$. 

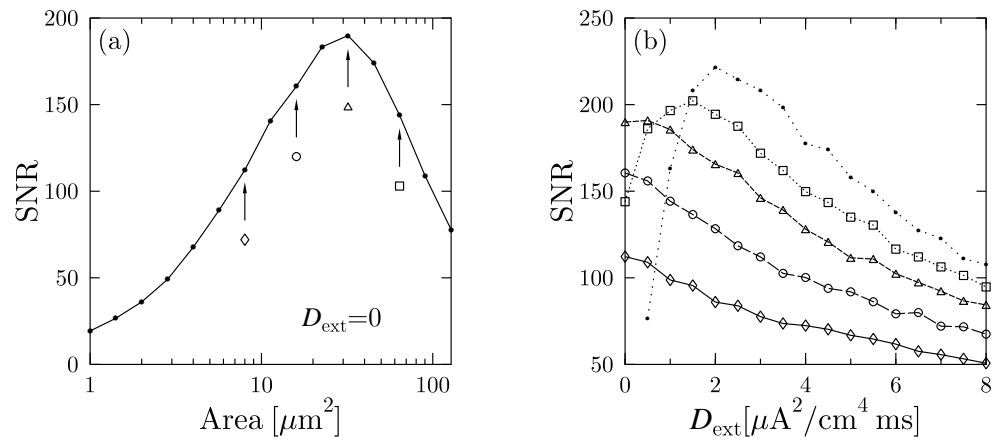

Figure 2: The signal-to-noise ratio (SNR) for an external sinusoidal stimulus with amplitude $A=1.0 \mu \mathrm{A} / \mathrm{cm}^{2}$ and frequency $\Omega=0.3 \mathrm{~ms}^{-1}$ for different observation areas: (a) No external noise is applied; (b) SNR versus the external noise for the system sizes indicated by the arrows in fig. 2(a). $S=8 \mu \mathrm{m}^{2}$ $\left(N_{\mathrm{K}}=144, N_{\mathrm{Na}}=480\right)$ : solid line through the diamonds, $S=16 \mu \mathrm{m}^{2}$ $\left(N_{\mathrm{K}}=288, N_{\mathrm{Na}}=960\right)$ : long dashed line connecting the circles, $S=32 \mu \mathrm{m}^{2}$ $\left(N_{\mathrm{K}}=576, N_{\mathrm{Na}}=1920\right):$ short dashed line through the triangles, $S=64 \mu \mathrm{m}^{2}$ $\left(N_{\mathrm{K}}=1152, N_{\mathrm{Na}}=3840\right)$ : dotted line connecting the squares. The situation with no internal noise (i.e., formally $S \rightarrow \infty$ ) is depicted by the dotted line.

For $S<32 \mu \mathrm{m}^{2}$ growing internal noise monotonically deteriorates the system response. Under such circumstances, one would predict that the addition of an external noise (which corresponds to the conventional situation in biological SR studies) cannot improve SNR further, i.e. conventional SR will not be exhibited. Our numerical simulations, fig. 2(b), fully confirms this prediction. Conventional stochastic resonance therefore occurs only for large membrane patches beyond optimal size, and reaches saturation in the limit $S \rightarrow \infty$ (limit of the deterministic Hodgkin-Huxley model). Thus, the observed biological SR [2, 3] is rooted in the collective properties of large ion channels arrays, where ion channels are globally coupled via the common membrane potential $V(t)$.

In conclusion, we have investigated stochastic and coherence resonance in a noisy generalization of the Hodgkin-Huxley model for excitable biological cell membrane patches. The spontaneous fluctuations of the membrane conductivity due to the individual channel dynamics has systematically been taken into account. We have shown that the excitable membrane patches with an observation area around $S \approx 1 \mu \mathrm{m}^{2}$ exhibit a rhythmic spiking activity optimized by everpresent internal noise. In other words, the collective dynamics of globally coupled ion channels become more ordered solely due to internal noise. This new effect can be regarded as the intrinsic coherence resonance phenomenon; it presents a first important result of our work. This very finding has also been confirmed independently within a different approach by Jung and Shuai [19] (see the accompanying paper). A second main result of this study refers to the phenomenon of intrinsic SR; thereby the internal noise alone gives rise to a SR 
behavior, see fig. 2(a). Conventional SR versus the external noise intensity takes place as well for sufficiently large membrane patches where the internal noise strength alone is not yet at its optimal value. We hence conclude that observed biological SR likely is rooted in the collective properties of globally coupled ion channel assemblies.

The authors gratefully acknowledge the support of this work by the Deutsche

Forschungsgemeischaft, SFB 486 Manipulation of matter on the nanoscale, Project A10. Moreover, we acknowledge most helpful and constructive discussions with Peter Jung.

\section{References}

[1] Gammaitoni L., Hänggi P., Jung P. and Marchesoni F., Rev. Mod. Phys. 70, 223 (1998).

[2] Douglass J. K., Wilkens L., Pantazelou E. and Moss F., Nature (London) 365, 337 (1993).

[3] Levin J. E. and Miller J. P., Nature (London) 380, 165 (1996).

[4] Collins J. J., Imhoff T. T. and Grigg P., Nature (London) 383770 (1996).

[5] Simonotto E., Riani M., Seife C., Roberts M., Twitty J. and Moss F., Phys. Rev. Lett. 78, 1186 (1997).

[6] Hidaka I., Nozaki D. and Yamamoto Y., Phys. Rev. Lett. 85, 3740 (2000).

[7] Bezrukov S. M. and Vodyanoy I., Nature (London) 378, 362 (1995); 385, 319 (1997).

[8] Goychuk I. and Hänggi P., Phys. Rev. E 61, 4272 (2000).

[9] Petracchi D., Pellegrini M., Pellegrino M., Barbi M. and Moss F., Biophys. J. 66, 1844 (1994).

[10] Hodgkin A. L. and Huxley A. F., J. Physiol. (London) 117, 500 (1952).

[11] Longtin A., J. Stat. Phys. 70, 309 (1993); Wiesenfeld K., Pierson D., Pantazelou E., Dames C. and Moss F., Phys. Rev. Lett. 72, 2125 (1994); Collins J. J., Chow C. C., Capela A. C. and Imhoff T. T., Phys. Rev. E 54, 5575 (1996); Lee S.-G. and Kim S., Phys. Rev. E 60, 826 (1999).

[12] Pikovsky A. S. and Kurths J., Phys. Rev. Lett. 78, 775 (1997); Lee S.G., Neiman A. and Kim S., Phys. Rev. E 57, 3292 (1998); Lindner B. and Schimansky-Geier L., Phys. Rev. E 61, 6103 (2000).

[13] Hille B., Ionic Channels of Excitable Membranes, 2nd ed. (Sinauer Associates, Sunderland, MA, 1992). 
[14] Nossal R. J. and Lecar H., Molecular and Cell Biophysics, (Addison-Wesley, Redwood City, 1991).

[15] Lowen S. B., Liebovitch L. S. and White J. A., Phys. Rev. E 59, 5970 (1999).

[16] White J. A., Rubinstein J. T. and Kay A. R., Trends Neurosci. 23, 131 (2000).

[17] Lecar H. and Nossal R., Biophys. J. 11, 1068 (1971).

[18] Clay J. R. and DeFelice L. J., Biophys. J. 42, 151 (1983); Strassberg A. F. and DeFelice L. J., Neural Comput. 5, 843 (1993); DeFelice L. J. and Isaac A., J. Stat. Phys. 70, 339 (1993).

[19] Jung P. and Shuai J., "Optimal sizes of ion channel clusters", preprint, (2001).

[20] Fox R. F. and Lu Y., Phys. Rev. E 49, 3421 (1994).

[21] Press W. H., Teukolsky S. A., Vetterling W. T. and Flannery B. P., Numerical Recipes in C, 2nd ed. (Cambridge University Press, 1992). 